\title{
Orientation of letters and their speed of recognition
}

\author{
PAUL A. KOLERS ${ }^{2}$ AND DAVID N. PERKINS \\ RESEARCH LABORATORY OF ELECTRONICS, MASSACHUSETTS INSTITUTE OF TECHNOLOGY
}

\begin{abstract}
In classical theories of perception, the visual experience is usually a passive reflection of the geometry of the stimulus. In modern "constructive" theories, the stimulus is a source of clues to an active problem-solving process in the visual system. Such theories require specification of what clues are important, and how they are used. This experiment required Ss to name inverted, reflected, and rotated alphabetic characters, scanning both from left to right and from right to left. Results show that neither direction of scan nor orientation of letters individually accounts for performance. but that their interaction does. Ss recognize letters by seeking clues to their identity within the context of the scanning motor sequence. The clues used the "elements" of recognition) do not correspond directly to formal aspects of the geometry of the letters.
\end{abstract}

In classical theories of visual pattern recognition, geometric and topological features of a stimulus are represented more or less passively in perception. Theories founded on template matching or on the passive uptake of geometric characteristics of the target are inadequate, however, to explain S's ability to cope with objects in unusual orientations or with the variety of shapes a general class such as "all letter As" might contain. A contemporary view is that $\mathrm{S}$ selects and abstracts aspects of the stimulus in a problem solving way to construct his perceptual experience; but neither the operations governing these constructive activities nor the "elements" upon which they are performed have been clearly specified. Some formulations suggest that the "elements" are geometric attributes of characters which are abstracted and somehow combined to build a representation which is then tested against the environmental sample (Kolers \& Eden, 1968; Neisser, 1967). This is a recursive sequence of operations that might be characterized as organize, test, reorganize, test, etc., and it contrasts with the classical unidirectional flow of information from the environment to S's memory.

Within this constructivist view the conceptual problems are twofold: to specify the characteristics of objects that people actually use in constructing their representations, and to specify the processes, rules, or algorithms that regulate the construction. No single algorithm can account for recognition, nor do we have any clear evidence about the way that particular processes are called into play. Here we report an investigation of some of these issues. We studied the effect of interfering with a normal way of processing alphabetic letters by altering their appearance and by altering the way they were scanned.

English text is normally scanned from left to right. The eyes move in a well-automatized motor sequence with little conscious attention from the reader. One function of this scanning is to adjust the reading material on the retina in sequential order, so that the visual system may perform its pattern recognition tasks and so that the reader may apply his other cognitive functions to making sense out of the material. The experiment discussed in this paper implies a more complex interaction between scanning and pattern recognition.

The experimental task required Ss to name geometrically transformed letters, strings of alphabetic characters that had been rotated, reflected, or inverted. In another paper (Kolers \& Perkins, in press), we discuss errors made in this experiment on ambiguous letters such as the set b,d,p,q. Both the patterning of the errors and the general success of Ss in coping with the texts are interpreted to show that Ss perceive the transformed letters within an "orientation set"; they keep track of the orientation of the text fairly well, and as they encounter each new letter are able to use their knowledge of its likely orientation to help recognize it. The suggestion was made that as well as having a general ability to deal with misoriented figures, Ss use specific constructive operations for building percepts in the various transformations.

In the experiment, Ss named pages of transformed letters both from left to right and from right to left. The natural supposition would be that on any given transformation the left-right scan, being better trained, would be more rapid. But the relation between performance time and scan direction was found to be more complex than this. The leftward scan, rather than being a new, clumsy, and uneducated skill, mirrors the rightward scan in its interaction with the transformed letters.

\section{METHOD}

The data were collected as part of the same experiment described by Kolers and Perkins (1969). Ten right-dominant male undergraduates, native speakers of English, named 16 pages of letters that had been transformed geometrically. The 16 pages formed two sets of eight pages each. In one set, Letters, every letter was followed by a blank space, 32 letters to the line, 26 lines to the page, or 832 letters. The second set, Pseudowords, preserved the distribution of word-lengths found in the page of connected discourse we used as source material; they were also 26 lines to the page, but the number of letters varied slightly from line to line, and there were about 1170 letters to the page. Both Letters and Pseudowords preserved the relative frequency of the letters in the source page.

Subjects were tested individually in two sessions. Half the Ss named the letters of Letters on the first test day and the letters of Pseudowords on the second; the other half worked in the reverse order. The Ss named the letters of four pages in each set from left to right and of the other four from right to left. The pages were encased in clear plastic envelopes and were placed in front of $S$ one at a time. An arrow indicated the direction in which the letters were to be named. Time and errors were recorded. The sequence in which the transformations were presented was only partially counterbalanced across Ss, but subsequent analysis revealed no significant effects of test order. ${ }^{3}$

Four examples of Letters and four of Pseudowords appear in Fig. 1; the asterisk shows where to begin naming each sample. The four transformations of Letters that are illustrated are rotations of lines of letters in three-dimensional space: normal appearance $(N)$, rotation in the plane of the page $(R)$, inversion (I), and mirror reflection (M). The four examples of Pseudowords repeat these transformations and add the transformation of reversing every letter individually on a vertical axis through itself: reversed normal $(\mathrm{rN})$, reversed rotation ( $\mathrm{rR}$ ), and so on. This notation, although less apt for single letters, is retained to maintain consistency with the notation for connected discourse (Kolers, 1968). The eight transformations therefore can be regarded as four pairs. Individually, the characters of $\mathrm{N}$ and $\mathrm{rM}$ are identical, as are $\mathrm{rI}$ and $R, r N$ and $M$, and $I$ and $r R$. The difference between the members of a pair is not their geometry but is the direction of naming.

Subjects use several strategies in this task. Some look at the 
N *bunlefotatoienotpiuishousweicesw

\author{
R те แе ч \\ I *
}

M b y

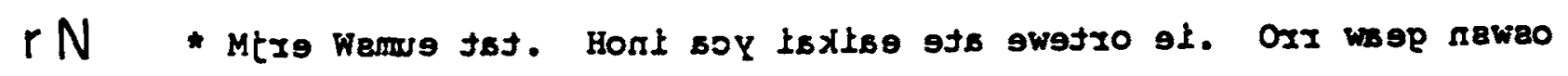

rR

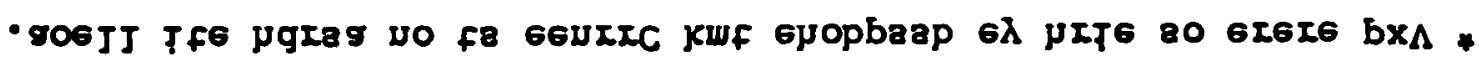

$r I$

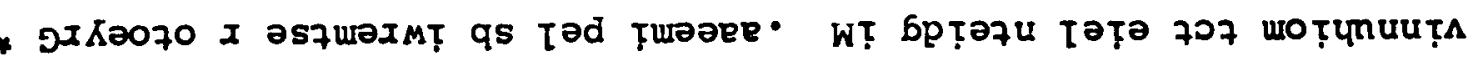

$r M$

itsfcrmbe iryouhhif aan you rasb eeo uuwncddr si pf gssue tgigk *

Fig. 1. Four examples of transformed letters as they appeared in Letters and in Pseudowords.

material silently for a while, some begin immediately to name the transformed letters, and others work in spurts of naming and looking. We used two tactics to compensate for this variability: First, Ss who did not begin to speak aloud within $10 \mathrm{sec}$ of seeing a page were then urged to begin, which all did; secondly, performance was timed beginning with S's speaking of the last letter on the first line of print. The data below, therefore, represent the speed of naming 800 letters in the Letters condition, the first 800 letters after the first line in Pseudowords, and the whole page of Pseudowords minus the first line.

\section{Speed in Naming}

\section{RESULTS}

Analysis of variance was carried out both on the measured times and on their logarithms. Obtained significance levels were identical in the two cases. The data shown in Table 1 are the arithmetic means of the measured time; they are arranged as pairs of transformations whose individual letters are identical in appearance. The upper member of each pair was named rightwards. In two comparisons, $\mathrm{N}$ and $\mathrm{rM}$, and $\mathrm{I}$ and $\mathrm{rR}$, speed is greater in the rightward direction of naming; in one comparison, $\mathrm{rN}$ and $\mathrm{M}$, the rightward direction is favored slightly; but in the fourth comparison, $\mathrm{rI}$ and $\mathrm{R}$, speed is greater in the leftward direction. The difference between $\mathrm{rN}$ and $M$ is statistically unreliable; the differences between the members of the other three pairs are significant below the 0.05 level in all three columns of the table. Thus, identical alphabetic characters require different amounts of time to be named, depending upon the direction of naming; however, direction interacts with the nature of the transformation imposed on the characters. Clearly, it is not the well learned skill of scanning rightward, by itself, that determines performance, nor can speed of naming identical characters be described independently of scanning direction.
Note that 800 characters named in 4.65 min represents a speech rate of $349 \mathrm{msec}$ per character. If Ss had had to compare every printed letter individually against some feature list in memory, the 52 comparisons (upper and lower case letters) would have had to occur rapidly enough to accommodate the 349-msec-per-character speech rate for $\mathrm{N}$, that is, at about $6.7 \mathrm{msec}$ per comparison. We know of no data that indicate that classification can occur so rapidly (cf. Sternberg, 1967).

These results hold for both Letters and Pseudowords; however, with the exception of $\mathrm{N}$, Ss took approximately 10 to $15 \%$ more time to name 800 letters of Pseudowords than 800 letters of Letters. The results show, therefore, that character recognition cannot be accounted for only in terms of the geometry of the characters, i.e., their hooks, bars, arches, and related features of their shapes. The density of the array (Letters vs Pseudowords), the direction of scanning, and the transformation imposed on the characters all affect performance.

Effect of Direction

The effect of direction interacts with transformations. The

Table 1

Average Time to Name Letters (minutes)

\begin{tabular}{cccc}
\hline Transformation & Letters & $\begin{array}{c}\text { 800 Letters in } \\
\text { Pseudowords }\end{array}$ & $\begin{array}{c}\text { Full pages of } \\
\text { Pseudowords }\end{array}$ \\
\hline N & 4.65 & 4.51 & 6.46 \\
IM & 5.66 & 6.16 & 9.14 \\
I & 7.96 & 8.77 & 12.76 \\
IR & 8.55 & 9.56 & 14.18 \\
IN & 7.06 & 7.86 & 11.59 \\
M & 7.20 & 8.04 & 11.33 \\
II & 7.16 & 8.33 & 12.17 \\
R & 6.72 & 7.64 & 10.87
\end{tabular}


Table 2

Order of Difficulty of Transformations*

\begin{tabular}{lllllllllll}
\hline Letters & $\mathrm{N}$ & $\mathrm{rM}$ & $\mathrm{R}$ & $\mathrm{rN}$ & $\mathrm{II}$ & $\mathrm{M}$ & $\mathrm{I}$ & $\mathrm{rR}$ \\
$\begin{array}{l}800 \text { letters } \\
\text { in } \mathrm{P}^{\prime} \text { words }\end{array}$ & $\mathrm{N}$ & $\mathrm{rM}$ & $\mathrm{R}$ & $\mathrm{rN}$ & $\mathrm{M}$ & $\mathrm{II}$ & $\mathrm{I}$ & $\mathrm{rR}$ \\
$\begin{array}{l}\text { Full pages } \\
\text { of } \mathrm{P}^{\prime} \text { words }\end{array}$ & $\mathrm{N}$ & $\mathrm{rM}$ & $\mathrm{R}$ & $\mathrm{M}$ & $\mathrm{rN}$ & $\mathrm{rI}$ & $\mathrm{I}$ & $\mathrm{rR}$
\end{tabular}

* Underlining indicates differences not significant at 5\% level.

interaction is not with transformation itself, however, but with a secondary attribute of transformation, particularly, the way the characters face. In some transformations the characters face to the right, as in $\mathrm{rM}$ and $\mathrm{I}$; in others they face left, as in $M$ and $R$. If $+F$ and $-F$ are used to indicate rightward or leftward facing of the characters, then characters in $N, I, r M$, and $r R$ can be designated $+F$, but performance on them is not always faster than on their $-\mathrm{F}$ mates $\mathrm{rN}, \mathrm{rI}, \mathrm{M}$, and $\mathrm{R}$. By itself, the way the character faces, and thus its familiar geometry, does not account for the results of Table 1. Therefore, neither direction of scanning by itself nor direction of facing by itself accounts for speed in naming; their combination does, however.

In some transformations the letters face in the direction they are named, but in others they do not. We will designate by $+\mathrm{C}$ and $-\mathrm{C}$ whether a character faced in the direction it was named or not. Then, characters in $N, I, M$, and $R$ can be designated as $+\mathrm{C}$; speed in naming these characters is equal to or greater than speed in naming their $-\mathrm{C}$ mates $r \mathrm{M}, \mathrm{rR}, \mathrm{rN}$, and $\mathrm{rI}$. S can usually perform faster with characters oriented in the direction in which he is scanning, independently of whether the direction is lefward or rightward. (Preliminary results suggest that the same holds true for letters oriented $90 \mathrm{deg}$ clockwise or counterclockwise to the normal position and named upward or downward.)

\section{Order of Difficulty}

The various transformations are not equally difficult; some induce more errors than others (Kolers \& Perkins, 1969) and some take more time than others. In Table 2 the transformations are arranged according to their order of difficulty, as inferred from the amount of time taken to name their letters. Differences that do not reach the 0.05 level of significance are shown by underlining. Overall, there is good agreement between Letters and Pseudowords. The order of difficulty of the simple transformations is $N, R, M, I$; the order of difficulty of those involving letter-reversal is $\mathrm{rM}, \mathrm{rN}-\mathrm{rI}, \mathrm{rR}$. This order is different from that found in connected discourse (Kolers, 1968).

\section{Construction of the Transformation Group}

The eight transformations of letters form an Abelian group (van der Waerden, 1953) that can be generated with many collections of three of the transformations. A question of great interest is whether performance on the set of eight transformations can be related to its geometrical construction. One way of asking the question is illustrated in Table 3, with the three basis elements, mirror reflection (M), inversion (I), and letter reversal (r). (Many other constructions of the group are possible; this one has been chosen for illustration.) The stars represent composition of transformations or geometric operators. The letters $\mathrm{M}, \mathrm{I}$, and $\mathrm{r}$ are the generating operators, $N$ is the identity operation, and the arrows show the results of their composition, the transformations illustrated in Fig. I. In parentheses next to each transformation is the difference in time needed to name those letters and to name the letters of $\mathrm{N}$ in the Letters condition (Table 1). The difference for $M$, for example, is $(M-N)=7.20-4.65=2.55$. Treating $N$ as a base line and subtracting it from performance on the other transformations leaves an increment in time that might be attributed to the work of decoding the transformation itself. If the visual system decoded the transformations by decoding their components serially, the sum of the increments of the components would equal the increment for the transformation. As illustration, if $R$ were decoded by applying the transformations I and $M$ serially, the sum of their increments would equal the increment for $\mathrm{R}$. It is obvious by inspection that this equality does not hold. Thus, the three operators we have chosen to illustrate the issue do not combine serially for the perceptual processing of the arrays. In the Appendix we prove that no set of three operators can fully account for the data, even in terms of parallel processing. The perceptual decoding of the transformations follows rules that are different from those governing their geometrical construction.

\section{DISCUSSION}

R zaders of English learn to read in the rightward direction. One might think, therefore, that naming letters from left to right would always be easier than naming them leftwards. The data of Table 1 contradict this belief. The well learned strategy of scanning rightward cannot by itself account for performance. The direction in which letters face also cannot by itself account for performance. Yet these two factors taken together do match the data. Naming is more rapid when the direction the letters face is the same as the scanning direction.

Therefore, the scan does not merely present data on a letter-by-letter basis to the perceptual mechanisms for their piecemeal analysis. Rather, there is a skilled sequencing or sweep to the naming of letter arrays, otherwise the direction of naming would have no influence on performance. Why is the consistency of direction of scan and facing of letters important? There are several possibilities.

One explanation can be put in the context of information-gathering strategies. The visual system must extract information from the stimulus in order to identify it. In the Roman alphabet in general, and especially in the type face used in this experiment (Fig. 1), the preponderance of their distinguishing features appear from the center to the right of letters. (Indeed, from discussions with type designers we have learned the interesting fact that the criterion for placing a letter in a design matrix is how well it looks in place with other letters. The criteria of acceptability are not geometric but perceptual.) A reasonable conjecture, then, is that the visual system normally samples a letter principally on its right. ${ }^{4}$

Now suppose this sampling was cued by the scanning direction. Then, when the direction the letter faced was different from the scanning direction, the visual system would tend to sample from an unaccustomed part of the letter. This would slow down the processing, either because the system would have to seek more information from the stimulus, or because the classification mechanisms would not be as well trained for the less familiar half of letters.

This explanation implies that only parts of letters are

Table 3

One Construction of the Transformation Group

$\mathrm{N} * \mathrm{M} \rightarrow \mathrm{M}$
$\mathrm{N} * \mathrm{I} \rightarrow \mathrm{I}$
$\mathrm{N} * \mathrm{I} \rightarrow \mathrm{rN}$
$\mathrm{N} * \mathrm{M} * \mathrm{I} \rightarrow \mathrm{R}$
$\mathrm{N} * \mathrm{M} * \mathrm{I} \rightarrow \mathrm{rM}$
$\mathrm{N} * \mathrm{I} * \mathrm{r} \rightarrow \mathrm{I}$
$\mathrm{N} * \mathrm{M} * \mathrm{I} * \mathrm{I} \rightarrow \mathrm{rR}$


examined in being recognized, the parts acting as clues for the skilled reader, and that these parts are sought within the context of a directional scanning strategy; after they are found, they are identified within the context of an orientation set.

The idea that characters are recognized within their transformations does not necessarily imply any priority to the decoding of the transformation. We specifically do not assert that $\mathrm{S}$ first decodes the transformation and then identifies the character within it. Sometimes the appearance of a character informs $S$ of the transformation imposed upon it, and sometimes the transformation defines the characters within it. The suggestion of an "orientation set" means that S need not compute the orientation of every letter or word, but can often carry over the orientation of the prior characters in a way that helps him to process a new character (Kolers \& Perkins, 1969).

There is no need to believe that the phenomenon being studied is due to one process alone or can be explained in only one way. Another possibility is this: coordinated automatized processes are often interrupted by any deviation in the expected sequence of events. The recognition of characters oriented in one direction might thus interfere with continuing a scan in the opposite direction. This interference would be suppressed by $S$, so that he might proceed, but the constant brief interruption of the motor process as $S$ had to scan a character in an unfamiliar way could lengthen the scanning and naming times. Conflicts of an apparently related kind characterize performance on Stroop's test, in which Ss are required to name the colors in which words are printed when the name and the color are discrepant, as when "red" is printed in blue. The discrepancy between color and color name impairs performance (Jensen \& Rohwer, 1966).

This explanation puts the interference in the general category of "mismatch reactions" which disrupt routine procedures. As such it is not specifically related to orientation set or to particular perceptual functions for dealing with misorientations. Another possibility, more closely tied to orientation set, is that the interference might not occur on such a general "interrupt" level, but might strike specifically at the orientation set itself. In other words, orientation set might be influenced both by the letters perceived and by the direction of scanning, so that a disagreement between the two would make maintaining an orientation set more difficult, and thereby slow the processing by making each encounter with a letter a new problem in orientation.

The visual system does not necessarily deal with only one letter at a time. Two or three letters may be perceived simultaneously although they are spoken sequentially. Scanning in a certain direction might form a set that the letters are ordered in that direction. If the letters faced in the opposite direction, this would interfere with the building of an organized percept of several letters at once, and hence might slow performance.

All these possibilities have a common feature. In all, the system is sensitive to the consistency between the motor movement (the scan of the eyes) and the arrangement of the target, both in transformation. Changing one requires a change in the other if smooth performance is to occur. The ability to deal with visual misorientations extends therefore to at least some visuomotor activities, even perhaps without special practice.

\section{Comparison with Reading}

The order of difficulty of the transformations is different for reading and for naming letters. This is a simple proof that the results with connected discourse (Kolers, 1968) cannot be accounted for only in terms of the difficulties in recognizing individual letters. There are some similarities in the ordering that are noteworthy, however.

The order of difficulty of the three-dimensional rotations is identical in the two tasks. On the early test days of the reading experiment the order was $\mathrm{N}, \mathrm{R}, \mathrm{M}, \mathrm{I}$-identical to the order shown in Table 2 for naming letters. (In the reading experiment maximum improvement occurred with I; thus, on later test days I was read more rapidly than M.) The major difference between the two tasks occurs with the transformations involving letter reversal. In reading, the order of difficulty was $\mathrm{rN}, \mathrm{rI}, \mathrm{rM}, \mathrm{rR}$; in naming letters, the order was $\mathrm{rM}, \mathrm{rN}-\mathrm{rl}, \mathrm{rR}$. Thus, the individual characters of $\mathrm{rM}$ are easily named; but collected as words, they are very difficult to read. Conversely, words in $\mathrm{rN}$ are read quite easily (more easily than $\mathrm{R}$, for example), but their letters are individually difficult to name. The overall similarity in the order of difficulty of the transformations for reading and character-naming preserves the hypothesis that the characters are recognized by means of perceptual operators (Kolers \& Perkins, 1969); the differences that do occur show in still another way that reading involves something else than the mere integration of a word's constituent letters.

\section{Characterization of Letters}

Our theory asserts that $S$ recognizes letters by selecting aspects of a visual array ("clues") that he constructs into a perceptual representation. In doing this, $S$ takes into account not only information from each letter, but also information from the total context, including orientation sets and scanning direction. Little is known, however, about the constructive processes that create the psychological representation of an object. One generative model, "analysis by synthesis" (Stevens \& Halle, 1967; Neisser, 1967), is seriously defective in its details (Kolers \& Eden, 1968). While, with Neisser, we assert that $S$ constructs a representation rather than merely detects a

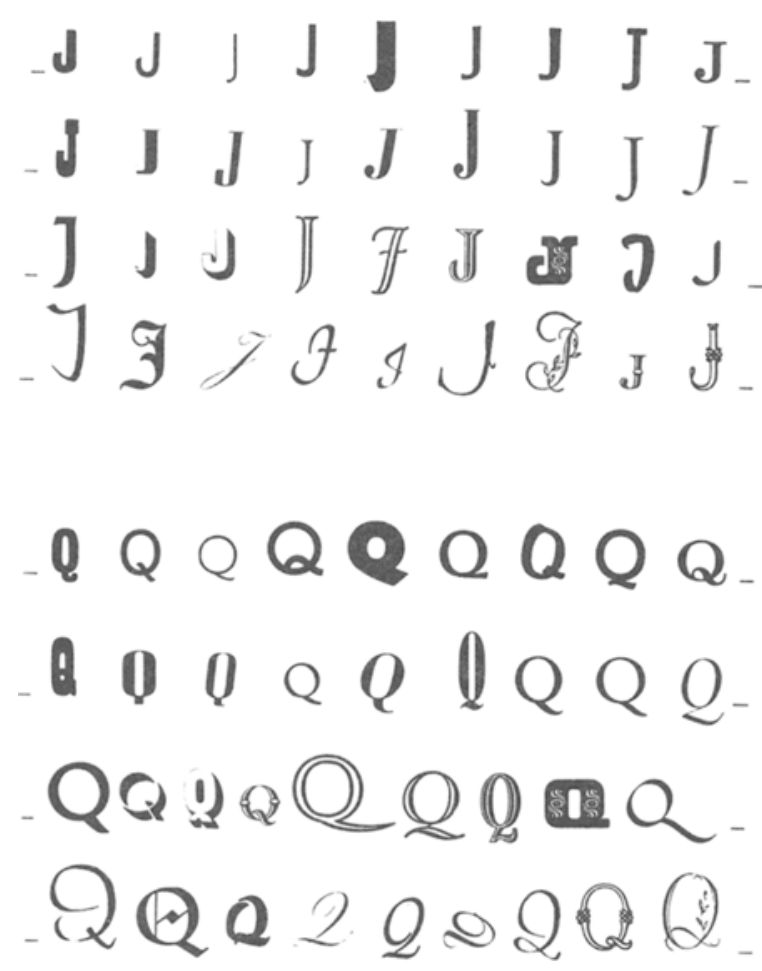

Fig. 2. Examples of the letters jay and kew in commercially available faces. The letters are normalized to a common base line, shown by the thin horizontals at the ends of the lines. 


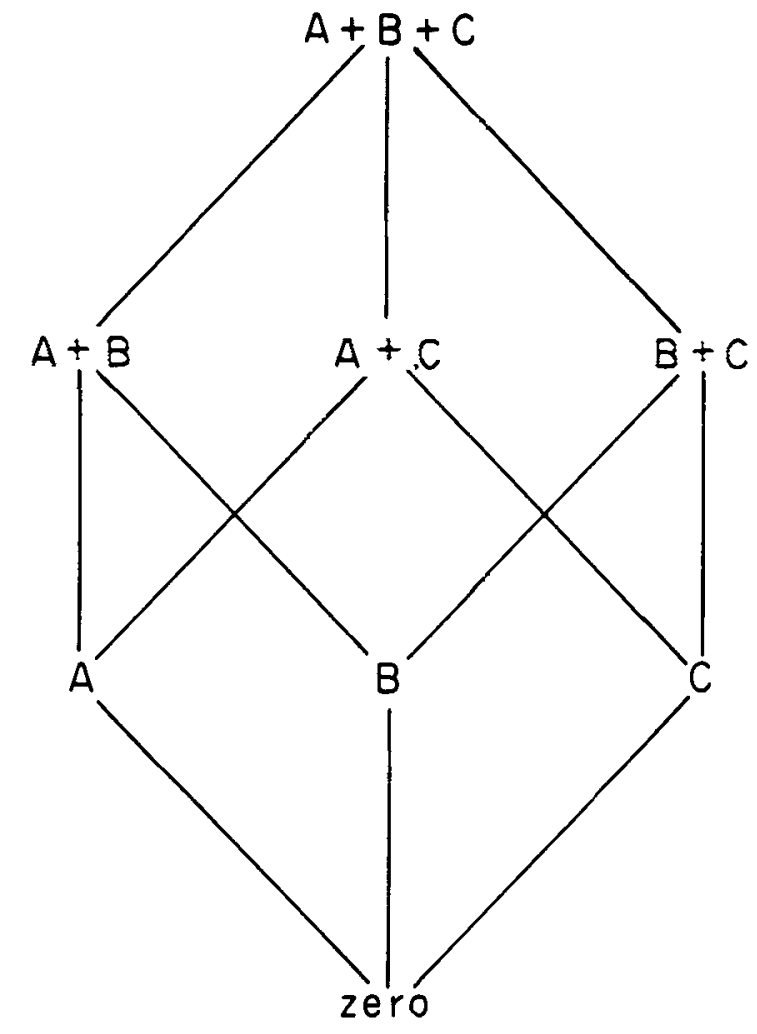

Fig. 3 A lattice illustrating the generation of eight transformations.

signal, it remains a fact that one cannot formally specify the aspects of a letter that $S$ uses in his construction.

Any letter of the English alphabet can be printed in an infinite number of ways. Any set of rules established to define a letter in terms of its geometry of hooks, bars, arches, loops, and the like can be violated easily and the letter still be recognized. In Fig. 2 are some instances of the letters jay and kew taken from a standard book of fonts (Anonymous, 1963). Note how inadequate template-matching, contour-tracing, or feature-listing theories are in respect to identifying the letters. It is within reason to conjecture that no deterministic principles can be established to define individual printed or written characters unambiguously. The rules can be drawn so as to minimize (or maximize) false positives and negatives, but never so as to make any imaginable instance of a letter recognizable. Thus, its specific geometry-the collocation of marks-cannot by itself provide a sufficient basis for a letter's recognition; hence, lists of "distinctive features" based on geometry cannot by themselves account for performance. What $\mathrm{S}$ uses as a clue to a letter's recognition may have little direct relation to the formally specifiable features of its geometry.

\section{APPENDIX}

We wish to show that S's coping with transformed letters does not correspond to the mathematical structure of the transformation group. Our argument is based on the order of difficulty of the transformations.

If a process, $A$, is to be ascribed even partly to a combination of subprocesses, $B$ and $C$, then certain time inequalities must hold. If $C$ is made up of $A$ and $B$, then $C$ should take at least as long as the longer of them. If strictly serial processing is involved, $C$ must be at least equal to the sum of $\mathrm{A}$ and $\mathrm{B}$; if some parallel processing occurs, $\mathrm{C}$ may be less than their sum. However, if $C$ takes less time than the longer of either $A$ or B, then some synergistic interaction is taking place, or A and B are not truly subprocesses of $C$. In either case, analyzing $C$ as independent subprocesses, $A$ and $B$, is improper.

We will call a monotone theory any hierarchy of processes in which a process takes at least as long as the longest of its subprocesses. We will call a strictly monotone theory any hierarchy of processes in which a process takes longer than the longest of its subprocesses. A strictly monotone theory is more restrictive than a monotone theory, for while not requiring that subprocesses be entirely sequential, it forbids that they be completely parallel.

The composition of transformations is fundamentally sequential, although, as the transformations form an Abelian group, no order of operations needs to be specified. Both a monotone and a strictly monotone theory, therefore, require fundamentally less than the mathematical structure itself; neither theory requires strict sequencing. Nevertheless, we will show that no strictly monotone theory based on three generators can satisfy the data of Tables 1 and 2 . One monotone theory, however, provides a rough approximation to the data.

There are 28 groups of three generators (i.e., $7 \times 6 \times 4 / 3$ !) whose composition yields the eight transformations studied. Any satisfactory triplet will produce the set of inequalities depicted in the lattice of Fig. 3, where the three are labeled A, B, C. For a strictly monotone theory, the time taken at any node of the lattice must be less than the time taken at the node above it. For a monotone theory, the time at the lower node must be less than or equal to the time at the upper. Note that the symbol "+" in Fig. 3 and in the text below is used not in its arithretic sense to indicate addition, but in its group theoretic sense to indicace any commutative operation.

Suppose that there is a monotone theory for the data. The order of difficulty of the transformations in Table 2 permits the following reasoning. $\mathrm{N}$ must occupy the lowest position in the lattice and $\mathrm{rR}$ the highest, for they require the least and the greatest amounts of time. Next after $\mathrm{N}$ is $\mathrm{rM}$; it must be either $\mathrm{A}, \mathrm{B}$, or $\mathrm{C}$ in Fig. 3. Say $\mathrm{rM}=\mathrm{A}$. Next find $B$ and $C$. $I$ cannot be $B$ or $C$ because $r M+I=r R$, which would then not be at the top of the lattice. I cannot be $A+B$ or $A+C$ because then $\mathrm{rR}$ would have to be $\mathrm{B}$ or $\mathrm{C}$, and it cannot be. Therefore, I must be $B+C$. Similarly, $r I$ cannot be $B$ or $C$ because $r M+r l=R$, but $R$ takes less time than $r I$. Therefore, $R$ must be $B$ or $C$; as it does not matter which, say $R=B$. Thus, we have found $0, A, B, A+B$, and $A+B+C$. These determine the entire lattice which is ilsustrated in Fig. 4.

Examination of the lattice shows that all of the lines indicate statistically significant differences in accordance with Table 2 with one exception. The strictly monotone theory requires that $M$ take less time than $\mathrm{rN}$; the monotone theory requires that $\mathrm{rN}$ be not longer than $\mathrm{M}$.

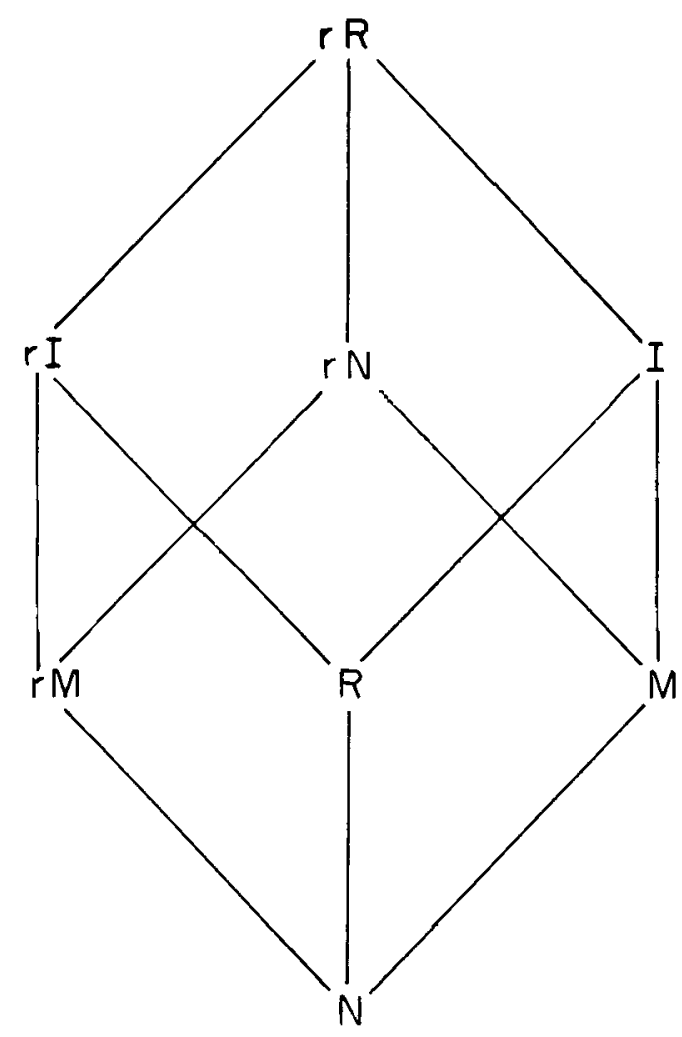

Fig. 4. The lattice that the data generate. 
Tables 1 and 2 reveal no statistically significant differences between $\mathrm{rN}$ and $\mathrm{M}$. Therefore, the strictly monotone theory-which is much weaker than the requirement that the transformations are decomposed perceptually in some form of serial processing-is rejected.

The following conclusions and implications summarize the proof: (1) A theory of serial decomposition of transformations corresponding to their mathematical structure derived from three basis elements is disproved. A weaker requirement, which permits some sharing of resources, the strictly monotone theory, is also disproved. (2) Only one monotone theory can approximate the data, one whose basis elements are $r M, R$, and $M$, but the fit is equivocal. The equivocation results from the failure of $r M$ to contribute tellingly to the processing time of $I N$.

Even if the monotone theory of basis elements $\mathrm{rM}, \mathrm{R}$, and $\mathrm{M}$ fitted the data better, it remains intuitively unappealing. One consequence of the theory is that $\mathrm{I}$ is decoded through $\mathrm{R}$ and $\mathrm{M}$, i.e., that an inversion is decoded by rotating a reflection or reflecting a rotation (the theory is indifferent to the order of operations). While a possible route for the nervous system to follow, this appears to be a particularly ungainly one.

Finally, despite the equivocation of the monotone theory, one may note the interesting fact that one of the required basis elements of the only possible monotone theory is $\mathrm{rM}$. In those transformations involving rM, Ss name letters in the direction opposite to the way they face. The discrepancy between direction of naming and orientation of the target was suggested in the body of the paper to be a critical feature of performance. That suggestion was based on still other considerations than those brought out in this Appendix.

\section{REFERENCES}

ANONYMOUS. The studio book of alphabets. New York: Viking, 1963. HUEY, E. B. The psychology and pedagogy of reading. New York: Macmillan, 1908. (Republished-Cambridge, Mass.: M.I.T. Press, 1968.)
JENSEN, A. R., \& ROHWER, W. D., JR. The Stroop color-word test: A review. Acta Psychologica, 1966, 25, 36-93.

KOLERS, P. A. The recognition of geometrically transformed text. Perception \& Psychophysics, 1968, 3, 57-64.

KOLERS, P. A., \& EDEN, M. (Eds.) Recognizing patterns: Studies in living and automatic systems. Cambridge, Mass.: M.I.T. Press, 1968. KOLERS, P. A., \& PERKINS, D. N. Orientation of letters and errors in their recognition. Perception \& Psychophysics, 1969, 5, 265-269.

NEISSER, U. Cognitive psychology. New York: Appleton-CenturyCrofts, 1967.

STERNBERG, S. Two operations in character recognition: Some evidence from reaction-time measurements. Perception \& Psychophysics, 1967, 2, 45-53.

STEVENS, $K$. N., \& HALLE, M. Remarks on analysis by synthesis and distinctive features. In W. Wathen-Dunn (Ed.), Models for the perception of speech and visual form. Cambridge, Mass.: M.I.T. Press, 1967.

van der WAERDEN, B. L. Modern algebra. New York: Ungar, 1953.

\section{NOTES}

1. This work was supported principally by the National Institutes of Health (Grants 1 P01 GM-14940-01 and 1 P01 GM-15006-01) and in part by the Joint Services Electronics Program (Contract DA 28-043-AMC02536 (E)). Some of the ideas discussed were worked out in Project Zero at the Harvard Graduate School of Education.

2. Address: Bell Telephone Laboratories, Holmdel, New Jersey 07733.

3. We thank Professor W. G. Cochran for advice on analyzing our incomplete Lat in square.

4. Some years ago Huey $(1908,1968)$ showed that in connected discourse the upper half of a line of print is more informative than the lower half. The relation seems to hold for individual letters also, but less well.

(Accepted for publication October 14, 1968.) 